2008;27:468-469). (Respond: David L Goldman MD, Division of Pediatric Infectious Diseases, Children's Hospital at Montefiore, Albert Einstein College of Medicine, 1300 Morris Park Ave, Bronx, NY 10461).

COMMENT. These findings in children confirm previous reports of a probable role for HSV-1 infection in adults with Bell's palsy. Acyclovir in treatment of Bell's palsy deserves further study. Other infectious causes reported include Epstein-Barr virus, mumps, enteroviruses, and rarely, varicella zoster virus (geniculate herpes, Ramsay Hunt syndrome).

\title{
INFLUENZA-ASSOCIATED ENCEPHALITIS/ENCEPHALOPATHY
}

The role of influenza A and influenza B in acute childhood encephalitis and encephalopathy (ACE) was evaluated prospectively in all children admitted to the Hospital for Sick Children, Toronto, Canada, during an 11-year period from Jan 1994- Dec 2004. Influenza infection was defined by detection in the nasopharynx by immunofluorescence microscopy or viral culture and/or by a 4 -fold or greater rise in complement fixation titer. In 311 children with ACE, influenza infection was detected in $22(7 \%)$; 11 were $<5$ years of age. Fourteen fulfilled criteria for ACE. Influenza A was detected in 13 of 14 cases, and influenza B in 1 case. Neurologic manifestations developed within 5 days of onset of respiratory symptoms in $64 \%$. These included seizures, cranial nerve abnormalities, focal motor deficits, gait abnormalities, meningismus, torticollis, hyperreflexia and opisthotonus. Two presented with status epilepticus, and 2 had hemiparesis. CSF pleocytosis occurred in 3 patients, and elevated protein in 4 . Neuroimaging abnormalities noted in 8 of 14 tested were more common in children $<2$ years of age. Neurologic sequelae occurred in 8 patients (in 5 $<2$ years of age), and included seizures, hemiparesis, ataxia, and speech disorder. EEGs were abnormal in all 8 of those with neurologic sequelae and in 4 of 6 without sequelae. An acute rather than a postinfectious process was suggested by the briefness of the respiratory prodrome. (Amin R, Ford-Jones E, Richardson SE, et al. Acute childhood encephalitis and encephalopathy associated with influenza. A prospective 11-year review. Pediatr Infect Dis J May 2008;27:380-395). (Respond: Ari Bitnun MD, FRCPC, University of Toronto, Division of Infectious Diseases, Hospital for Sick Children, 555 University Ave, Toronto, Ontario, Canada M5G 1X8. E-mail:ari.bitnun@sickkids.ca).

COMMENT. Influenza virus infection is associated with $5 \%$ of cases of acute childhood encephalitis/encephalopathy in Canada. The younger children $<5$ years of age are most susceptible, and children $<2$ years of age are most likely to have neurologic sequelae.

A Japanese study of prognostic factors in influenza-associated encephalopathy evaluated 442 cases retrospectively. (Nagao T, Morishima T, Kimura $\mathrm{H}$ et al. Pediatr Infect Dis J 2008;27:384-389). Type A influenza was detected in $84 \%$ and type B in $9.5 \%$. Fifty-four cases $(22 \%)$ had a history of febrile convulsions. Significant factors for a poor prognosis and death in $35(19 \%)$ of 184 cases were an elevated transaminase, hyperglycemia, hematuria or proteinuria, and use of diclofenac sodium for fever during the infection. Factors showing a trend toward poor prognosis were elevated body temperature $(>41 \mathrm{C})$, low platelets, and low blood sugar. The occurrence of these signs should prompt admission to intensive care. 